\title{
EL PROGRAMA CITI: UNA ALTERNATIVA PARA LA CAPACITACIÓN EN ÉTICA DE LA INVESTIGACIÓN EN AMÉRICA LATINA
}

\author{
Sergio Litewka*, Kenneth Goodman** y Paul Braunschweiger***
}

\begin{abstract}
Resumen: El Programa CITI (Collaborative Institutional Training Initiative) es un curso basado en Internet y desarrollado por voluntarios, que brinda material educacional sobre protección de sujetos humanos en investigaciones biomédicas y de conducta, buenas prácticas clínicas, conducta responsable de la investigación y trato humanitario a animales de laboratorio. Es un esfuerzo conjunto del Fred Hutchinson Cancer Research Center y la Universidad de Miami.

Con la colaboración de expertos locales, gran parte del contenido se ha adaptado para América Latina, se ha traducido al español y portugués y se han establecido acuerdos de colaboración con instituciones especializadas en bioética en Chile, Brasil, Costa Rica y Perú. Los autores presentan una metodología accesible para la capacitación en ética de la investigación y una justificación de su uso y difusión en América Latina
\end{abstract}

Palabras clave: Programa CITI, protección de sujetos humanos, América Latina, ética de la investigación

\section{THE CITI PROGRAM: AN ALTERNATIVE TO TRAIN LATINAMERICA IN INVESTIGATION'S ETHICS}

Abstract: The CITI Program (Collaborative Institutional Training Initiative) is a course based on Internet with no profit intention; it has been developed by volunteers who offer educational subjects on human beings' protection in biomedical investigations, as well as on conduct, good medical practices, responsible conduct in investigation and humanitarian behaviour towards laboratory animals. This Program is a joint effort done by the Fred Hutchinson Cancer Research Centre and the University of Miami.

With the collaboration of local experts a considerable part of its content has been adapted for Latin America, it has been translated to Spanish and Portuguese and collaborative agrees have been established with bioethics' specialized institutions in Chile, Brazil, Costa Rica and Peru. Its authors present an accessible methodology in order to train on investigation's ethics and to justify its use and spreading out throughout Latin America.

Key words: CITI Program, human beings' protection, Spanish, Portuguese, Latin America, investigation's ethics

\section{O PROGRAMA CITI: UMA ALTERNATIVA PARA A CAPACITAÇÁO NA ÉTICA DA PESQUISA NA AMÉRICA LATINA}

Resumo: O programa CITI (Iniciativa de treinamento institucional colaborativo) é um curso baseado na internet e desenvolvido por voluntários, que fornece material educacional sobre a proteção de sujeitos humanos nas pesquisas biomédicas e de conduta, boas práticas clínicas, conduta responsável da pesquisa e trato humanitário para animais em laboratório. É um esforço conjunto do Fred Hutchinson Câncer Research Center e a Universidade de Miami.

Com a colaboração de peritos locais, grande parte do conteúdo foi adaptado para a América Latina e traduzido para o espanhol e português e se estabeleceram acordos de colaboração com instituiçôes especializadas em bioética no Chile, Brasil,Costa Rica e, Peru. Os autores apresentam uma metodologia acessível para a capacitação em ética da pesquisa e uma justificação de seu uso e difusão na América Latina.

Palavras chave: Programa CITI, proteção dos sujeitos humanos, América Latina, ética da pesquisa

* Profesor del Programa de Bioética de la Universidad de Miami. Coordinador del Programa CITI para América Latina, Estados Unidos

** Director del Programa de Bioética de la Universidad de Miami. Miembro de la Junta Directiva del Programa CITI, Estados Unidos

*** Profesor de Radiación Oncológica, Universidad de Miami. Director de la Oficina de Educación y Entrenamiento para la Investigación de la Universidad de Miami. Cofundador del Programa CITI, Estados Unidos

Correspondencia: slitewka@med.miami.edu 
Sin principios éticos no hay investigación posible Sin investigación no hay salud Sin salud no hay desarrollo Ken Goodman y Anthony Mullings ${ }^{1}$

\section{Introducción}

La inversión que los países de América Latina y el Caribe dedican a ciencia y tecnología, como porcentaje de su Producto Interno Bruto (PIB), está muy por debajo del gasto en el mismo rubro por parte de sus iguales de América del Norte, Europa, Oceanía y algunos países de Asia.

De acuerdo con un documento reciente de UNESCO"(1), el promedio del PIB que los países de América Latina y el Caribe dedican al desarrollo de la investigación científica en la región es de alrededor del $0,4 \%$, con excepción de Brasil, en donde llegaría a casi el $1 \%$.

Esta falta de interés en el desarrollo de programas de investigación y desarrollo científico se acompaña de numerosas falencias en programas para la educación y capacitación de científicos en los aspectos clave de la ética de investigación con seres humanos. Al mismo tiempo, las empresas farmacéuticas han incrementando exponencialmente su participación en ensayos clínicos en América Latina y el Caribe.

Según reportes publicados por las llamadas Organizaciones de Investigación por Contrato, o CRO por sus siglas en ingles: "América Latina ha probado ser un jugador mayor en la industria (farmacéutica) con ventajas significativas en lo que respecta a sus facilidades y recursos..."(2).

Con una población de 40 millones de personas en Argentina, 190 millones en Brasil y 108 millones en México, y con sistemas de salud dispares en cuanto a accesibilidad y calidad, pero que en muchos centros urbanos ofrecen características similares a las de países desarrollados, es perfectamente entendible que América Latina se haya convertido en un sitio atractivo para la industria farmacológica(3).

El impacto positivo que la investigación biomédica genera, tanto en la economía de las organizaciones

1 Coordinador de CITI para el Caribe angloparlante. Departamento de Obstetricia de la Universidad de las Indias Occidentales, Kingston, Jamaica. de salud como en el prestigio de quienes trabajan en ellas, muchas veces se ve empañado por las dificultades para brindar salvaguardas a los seres humanos que son objeto de estudio(4-6).

Numerosos autores coinciden en las dificultades que atraviesa la región para igualar los estándares éticos de los países de los cuales son originarias las empresas patrocinantes. Se menciona la falta o ineficacia de los cuerpos reguladores, las disparidades en la revisión de los protocolos por parte de los comités de ética de investigación (CEI), los problemas para conseguir quórum en las reuniones de los comités y mantener adecuadamente los registros correspondientes, la ausencia de una metodología y pautas para obtener consentimiento informado, dificultades que se magnifican ante la escasez de programas de educación sobre los aspectos éticos de la investigación con humanos(7-11).

Las consecuencias ponen en riesgo tanto la calidad de los estudios como de los investigadores e instituciones, originando una profunda desconfianza en la sociedad acerca de los eventuales beneficios de los ensayos clínicos con seres humanos(12).

Desde hace más de una década, el Programa de Bioética de la Organización Panamericana de la Salud, con sede en Santiago de Chile, ha sido pionero en el desarrollo de modelos educativos en ética de la investigación. El Programa CITI, un modelo de enseñanza que utiliza Internet, es complementario a los esfuerzos ya iniciados, siendo una herramienta accesible para los investigadores, miembros de comités de ética y todos aquellos vinculados a la experimentación con seres humanos.

\section{Breve descripción histórica del Programa CITI}

El Programa de Educación en Ética de la Investigación o, por sus siglas originales en inglés, Collaborative Institutional Training Initiative (CITI $)^{2}$, fue concebido en junio de 2000 como una respuesta a un requisito formulado por el Departamento de Salud y Servicios Humanos de Estados Unidos de América, el que exige que, en forma previa a desarrollar cualquier tipo de investigación con seres humanos, debe aprobarse un

2 Sitio en Internet: www.citiprogram.org 
curso de capacitación sobre las pautas éticas elementales para llevar a cabo la misma.

En los comienzos del Programa, tres representantes de diez organizaciones con experiencia en el desarrollo de programas de protección de sujetos humanos aceptaron la propuesta de los cofundadores de CITI -Karen Hansen, directora de la Oficina de Revisión Institucional del Instituto Fred Hutchinson, en Seattle, y Paul Braunschweiger, profesor del Departamento de Irradiación para Patologías Oncológicas y director de la Oficina de Educación y Entrenamiento para la Investigación de la Universidad de Miami- de formular un sistema de aprendizaje accesible, oportuno y con sólidos fundamentos teóricos en aspectos éticos y regulatorios para la participación de seres humanos en estudios biomédicos y de conducta.

La visión inicial de los fundadores era crear un modelo educativo adaptable a las necesidades de los cursantes, entendiendo que éstos provendrían de disciplinas diversas y con distintos roles en las etapas de la investigación científica.

De esta forma, se desarrollaron los primeros textos los que, una vez concluidos, se presentaron a revisión por pares. Una vez que fueron aprobados, se incorporaron al software utilizado por la Universidad de Miami para sus cursos de enseñanza a distancia. Además, diez organizaciones de Estados Unidos, entre las cuales se encontraban la Asociación de Psicólogos, la Universidad de Columbia, la Universidad del Estado de Mississippi, la Universidad de Virginia, el Departamento Correccional de Carolina del Norte y la Universidad de Chicago, fueron pioneras al aceptar este nuevo modelo de aprendizaje y certificación.

El crecimiento de CITI excedió las expectativas iniciales y trascendió las fronteras de Estados Unidos, por lo que, respondiendo a los pedidos, se creó www.irbtraining. org, una plataforma internacional de acceso libre con una serie de capítulos sobre protección de sujetos humanos. Los contenidos iniciales se tradujeron al español, chino, portugués, francés y urdu.

Se estableció que los nuevos contenidos se revisarían en encuentros semianuales. En cada uno de éstos, los desarrolladores ampliaron y refinaron el material didáctico, y se agregó un curso de repaso para aquellas organizaciones que necesitaban recertificar a sus investigadores. Esta iniciativa fue liderada por Lorna
Hicks, directora del Programa para Protección de los Sujetos Humanos en Investigaciones No-Médicas de la Universidad Duke.

A medida que nuevas universidades se agregaban a la lista de desarrolladores, el Departamento de Hospitales de Veteranos de Estados Unidos, con la colaboración de Mike Fallon y la participación de Paul Braunschweiger, desarrolló un nuevo software, simplificando el acceso, control y certificación de los cursantes del Programa CITI.

Al curso inicial sobre protección de sujetos humanos se han agregado 15 módulos sobre Buenas Prácticas Clínicas, de especial utilidad para investigadores que trabajan en proyectos globales. Sus versiones originales han sido adaptadas y traducidas al español y portugués. Se ha desarrollado un contenido independiente sobre manejo de la información y confidencialidad, preparado por tres expertos en la materia: Reid Cushman, del Departamento de Tecnología de la Información de la Universidad de Miami; Kenneth Goodman, director del Programa de Bioética de la Universidad de Miami, y Anita Cava, codirectora del mismo.

Finalmente, se incorporaron módulos sobre conducta responsable en la investigación. Las versiones originales fueron creadas por autores estadounidenses y, recientemente, se agregaron capítulos originales en español desarrollados por Eduardo Rodríguez y Fernando Lolas, del Centro Interdisciplinario de Estudios en Bioética (CIEB) de la Universidad de Chile.

El último contenido que ha sido integrado está dedicado al trato humanitario de animales de laboratorio.

\section{Relevancia de la enseńanza de los aspectos éticos de la investigación con seres humanos en América Latina}

Todas las sociedades enfrentan desafíos cuando desarrollan investigaciones científicas. Por cierto, la supervisión ética adecuada y oportuna no es un asunto menor. Numerosas situaciones irregulares generadas en estudios biomédicos llevados a cabo en América Latina y patrocinados por empresas internacionales han tenido repercusión internacional(13). Las respuestas por parte de las autoridades locales, cuando existieron, fueron nuevas regulaciones que se superponen a las existentes, creando un confuso panorama de disposiciones nacionales, provinciales, municipales, además 
de aquellas que ya están contempladas en los colegios profesionales de las organizaciones científicas(14). Esta discordancia pone en evidencia que las regulaciones por sí mismas no son suficientes para reemplazar el conocimiento bioético.

Coincidimos con Lolas en que se debe distinguir entre legalidad y legitimidad en el análisis ético de la investigación clínica: "Legalidad se refiere al cumplimiento de procedimientos aceptados por la comunidad científica relativos a la validez, confiabilidad y solvencia del trabajo de investigación. Legitimidad alude a la propiedad con que en el contenido de la publicación (o estudio) se respetan los principios éticos(15).

Si los programas de educación en ética de la investigación son escasos, el uso de Internet como herramienta de capacitación es un hecho novedoso y merece ser considerado. Sin que existan datos concluyentes de su eficacia, puede afirmarse que algunas de sus ventajas potenciales son evidentes: economiza tiempo y recursos financieros a la vez que permite el acceso a número significativo de personas de manera simultánea durante las 24 horas y los siete días de la semana.

El Programa CITI desarrolló cuestionarios para los cursantes (todos aprobados previamente por el CEI de la Universidad de Miami) tendientes a evaluar la aceptabilidad en sus currículos de la metodología utilizada, en contraposición con los modelos habituales de educación. El objetivo de estas encuestas fue medir el impacto que los cursos han tenido en aspectos vinculados con la protección de sujetos humanos y la conducta responsable en la investigación.

Si bien resultaría ingenuo pretender que las conductas dolosas se modificarán por tomar un curso on line, las respuestas permiten suponer que el conocimiento de las fundamentos bioéticos, en conjunto con el de las normas existentes, sienta las bases para un mejor desempeño por parte de los investigadores(16).

Otros cuestionarios se concentran en la aceptabilidad del programa CITI, comparando las posibilidades que ofrece Internet con las diferentes opciones existentes. Todas las encuestas han sido anónimas y voluntarias. Como se observa en la figura 1, quienes han respondido han privilegiado la metodología desarrollada por CITI respecto de otras alternativas. Tal vez sea prematuro pensar en un cambio de paradigma, ya que sólo han respondido 1.132 usuarios, pero los resultados per- miten suponer que un programa basado en la WEB representa una solución aceptable.

Figura 1: Aceptabilidad del sistema utilizado

Asumiendo un compromiso que le ocupe la misma cantidad de tiempo, ¿cuál de las siguientes opciones preferirían en vez de Internet? $\mathrm{n}=1132$

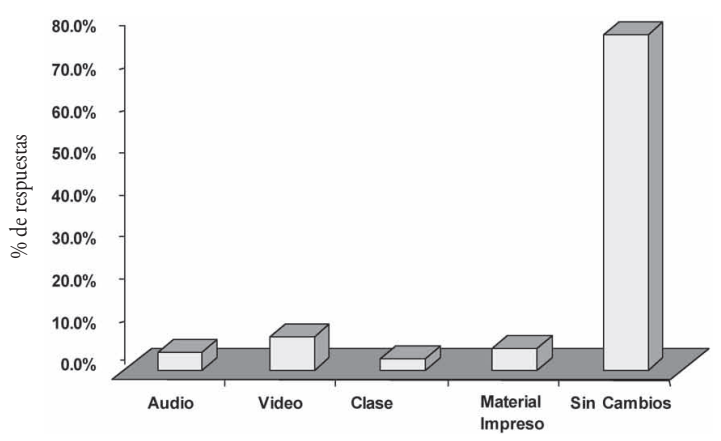

Otro cuestionario estuvo orientado a determinar el interés por parte de los cursantes en expandir el Programa hacia módulos con contenido sobre conducta responsable en la investigación, mala conducta científica, relaciones entre mentores y estudiantes, revisión por pares y manejo responsable de los datos de la investigación, considerando confidencialidad y privacidad. En este caso respondieron 2.691 cursantes y, de acuerdo con estas respuestas, se agregó nuevo contenido considerando los temas mencionados.

Figura 2: Nuevo contenido para el Programa CITI

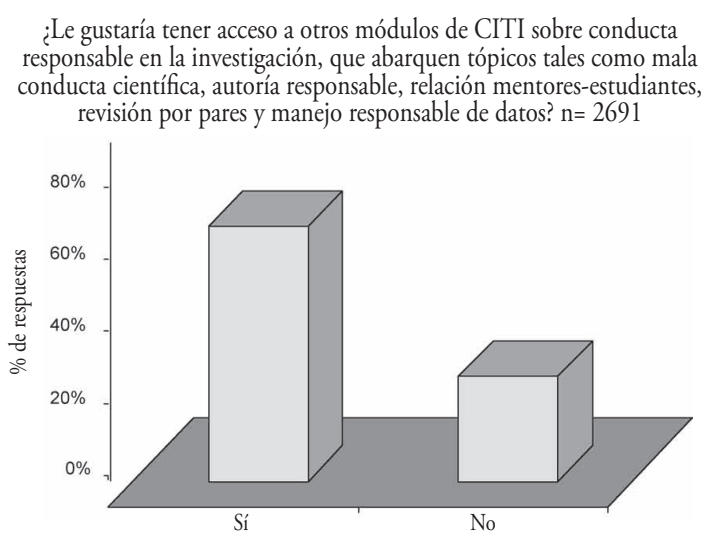

Todos estos cursos han sido agregados a CITI con posterioridad a la encuesta.

\section{El Programa CITI en América Latina}

$\mathrm{Al}$ igual que Internet, la investigación es un fenómeno global. La colaboración internacional es un proceso complejo que requiere minimizar los riesgos para los 
sujetos, asegurando que se cumplan los estándares internacionales en cuanto a la protección de las personas. Los módulos del Programa CITI sobre la metodología para la obtención y el seguimiento del consentimiento informado, el respeto a los sujetos de investigación, la beneficencia, la privacidad y la confidencialidad en el manejo de la información, los recaudos al llevar a cabo investigaciones con menores, mujeres embarazadas o poblaciones vulnerables no pretenden ser una injerencia cultural sobre otros países, sino que intentan reflejar estos elementales valores universales sin descuidar los aspectos sociales, culturales y legales que son particulares a cada nación de América Latina.

Por tal motivo, previo al inicio de nuestras actividades en la región, solicitamos la colaboración de especialistas en bioética oriundos de América Latina. Hasta el momento, el Programa CITI ha recibido el aporte, sea por traducciones y adaptaciones, o bien mediante la creación de nuevos módulos específicos, por parte del ya mencionado Centro Interdisciplinario de Estudios en Bioética de la Universidad de Chile.

Otros nuevos desarrolladores provienen de la Universidad de Caxias do Sul y la Fundación de Amparo a la Investigación del Estado de Minas Gerais, ambas de Brasil, y del Centro Médico de Investigaciones de la Armada de los Estados Unidos (NMRCD por sus siglas en inglés) en Perú. Respecto de esta última organización, la certificación de CITI resulta obligatoria para todas aquellas personas involucradas en investigaciones biomédicas que se desarrollan bajo el patrocinio de NMRCD.

Hasta el presente, cerca de 800 personas provenientes de América Latina se han inscrito en los cursos ofrecidos por CITI. De éstas, 742 provenían de Perúz, en tanto el resto eran originarios de Ecuador, Venezuela, Guatemala, Argentina y Uruguay. Casi todos los inscritos tenían algún tipo de vínculo con los ministerios de salud locales.

Globalmente, 825 organizaciones forman parte de la red CITI. De ese total, unas 20.000 personas completan los cursos cada mes, en tanto cada semana se suscriben, en promedio, tres nuevas instituciones, cuyos integrantes, luego de completar los módulos que les son

3 CITI desarrolló una presentación del Programa durante un simposio auspiciado por NMRCD, la Universidad de Washington y el Instituto Nacional de Salud de los Estados Unidos, el que se llevó a cabo en las ciudades de Lima y Arequipa (Perú) durante mayo de 2007. asignados por los responsables de cada organización, reciben el certificado correspondiente.

\section{Adaptabilidad del Programa CITI}

La flexibilidad de CITI permite que los responsables de cada organización que se integra al curso adapten el contenido a las necesidades de formación de sus integrantes, dependiendo de la función que desempeñan en la investigación. De tal forma, el Programa permite que los organizadores locales integren sus materiales en un currículo adaptable, provean a sus administradores las herramientas para hacer el seguimiento de las actividades de sus cursantes y entreguen el certificado de finalización, una vez que los requisitos establecidos por la organización se han cumplido.

Actualmente el programa CITI cuenta con los siguientes contenidos:

A- Cursos básicos sobre protección de los sujetos humanos.

- Con enfoque biomédico: 12 módulos.

- Con enfoque hacia las ciencias sociales y de conducta: 11 módulos.

- De interés general: 4 módulos.

- Con contenido no centrado en Estados Unidos: 18 módulos.

- 32 Módulos sobre Protección de Sujetos Humanos (en español) y varios más en proceso de revisión.

B- Buenas Prácticas Clínicas (BPC) para investigadores:15 módulos (traducidos al español y portugués).

C- Cursos para la Conducta Responsable en la Investigación.

- Más de 50 módulos y estudios de caso para investigadores en ciencias sociales y de conducta.

- Manejo de la información, confidencialidad y privacidad: 18 módulos.

D- Uso de animales de laboratorio en experimentos: 16 módulos.

A modo de ejemplo, en la tabla 1 se mencionan algunos de los títulos disponibles en español sobre protección de sujetos humanos en investigaciones biomédicas que forman parte del contenido internacional de CITI y que son de interés para América Latina 
Tabla 1

Estudiantes que investigan y estudiantes que son sujetos en investigaciones.

Historia de la ética de la investigación biomédica, sus principios y su aplicación a los sujetos humanos de investigación.

Regulaciones y procesos básicos en la revisión del Comité de Ética de Investigación.

Pautas de CIOMS - 2002.

Comités de ética de investigación: autoridad y rol en la revisión de los protocolos de investigación.

Ensayos clínicos en América Latina.

Aspectos internacionales en consentimiento informado.

Evaluación de riesgo en investigaciones en ciencias sociales y del comportamiento.

Privacidad y confidencialidad.

Investigaciones con datos de archivo.

Investigaciones sociales y del comportamiento. Aspectos éticos para investigadores biomédicos.

Investigación con tecnología genética.

Investigaciones con poblaciones protegidas. Sujetos vulnerables: una definición

Investigación con presos.

Investigación con menores.

Investigaciones con mujeres en edad fértil y embarazadas, e investigaciones con fetos.

Daño a grupos. Investigaciones con poblaciones cultural o médicamente vulnerables.

Trabajadores como sujetos de investigación: una población vulnerable.

Investigación regulada por la FDA y Conferencia Sobre la Armonización (ICH).

Investigación internacional.

Investigación utilizando Internet.

Conflictos de interés en investigaciones con sujetos humanos.

Ética en la publicación de resultados de investigaciones biomédicas.

Ética de la investigación en la República Argentina.

Regulaciones para la investigación biomédica en Chile.

Aspectos éticos en estudios con poblaciones especiales en América Latina.

Investigación con mujeres. Una perspectiva desde América Latina.

Investigación con animales.

Aspectos éticos de la investigación con vacunas (en proceso de publicación).

Integridad en la investigación científica (en proceso de publicación).

Al finalizar cada capítulo, el cursante debe completar un cuestionario de comprensión, el cual, una vez aprobado, le permite acceder al siguiente módulo. Los coordinadores locales de cada institución son quienes deciden los tópicos obligatorios y los optativos, de acuerdo con la función que desempeñan sus integrantes en la investigación. Asimismo, los responsables locales determinan el puntaje mínimo necesario para aprobar cada uno de los temas tratados. De esta manera, CITI descentraliza las decisiones, permitiendo que cada organización pueda manejarse de acuerdo a sus intereses y necesidades.

\section{Hardware y soporte tecnológico}

El Programa CITI está hospedado en un centro de base de datos SQL con un servidor de soporte y otros cuatro servidores de la red localizados en el Departamento de Información Tecnológica de la Universidad de Miami. El curso está configurado de tal manera que permite hasta 2.000 cursantes simultáneos sin que esta carga afecte el tiempo de respuesta. El servidor principal
SQL puede ser rápidamente reemplazado en caso de ser necesario.

Asimismo, existe un centro de apoyo en la Universidad de Miami que brinda ayuda en idioma español y portugués para los cursantes provenientes de América Latina.

\section{Conclusiones}

La participación de los países de América Latina en las investigaciones biomédicas, especialmente en el área de la farmacología, está creciendo exponencialmente. Aunque en forma dispar, las naciones del hemisferio han establecido distintos marcos regulatorios para asegurar la protección a los seres humanos que son objeto de experimentación. Estos marcos deben complementarse con el conocimiento de los fundamentos éticos de la investigación científica.

El Programa CITI pretende ser una herramienta accesible para el mayor número posible de personas, a la vez que una fuente de intercambio de conocimiento con 
los especialistas locales. Este intercambio permite:

- Compartir principios y objetivos para mejorar los componentes éticos en la investigación con seres humanos.

- Promover una cooperación internacional que genere nuevo material de estudio, en español y portugués, basado en las pautas internacionales y sensible a la problemática local.
- Contribuir a mejorar la calidad de la investigación científica.

Los nuevos contenidos para América Latina seguirán siendo desarrollados y evaluados en conjunto con especialistas de la región, pioneros en el desarrollo de programas de educación en ética de la investigación en el hemisferio.

\section{Referencias}

1. UNESCO. Latin América is just not getting it together. Science Policy and Sustainable Development 2006. (Sitio en Internet) Disponible en http://www.unesco.org/science/psd/publications/rep_lac_05.shtml Último Acceso en enero 29 de 2008.

2. Chu W. SIPLAS sign agreement for clinical trial support. DrugResearcher.com, 16 Febrero 2006. (Sitio en Internet) Disponible en http://www.drugresearcher.com/news/ng.asp?id=65878 Último Acceso en enero 29 de 2008.

3. Barnes K. Parexel talks clinical research in Latin America. PharmaTechnologist.Com, Diciembre 7, 2007. (Sitio en Internet) Disponible en http://www.in-pharmatechnologist.com/news/ng.asp?id=78007 Último Acceso en enero 29 de 2008.

4. Hyder A, Wali S, Kahn A. Ethical review of health research: A perspective from developing countries' researchers. Journal of Medical Ethics 2004; 30: 68-72.

5. Caniza MA, Clara W, Maron G, et al. Establishment of ethical oversight of human research in El Salvador: Lessons learned. The Lancet 2006; 7: 1027-1033.

6. Lavery J. Putting international research ethics guidelines to work for the benefit of developing countries. Yale Journal of Health Policy, Law and Ethics 2004; 4: 319-336

7. Rivera R, Ezcurra E. Composition and operation of selected research ethics review committees in Latin America. IRB: Ethics and Human Research 2001 September-October; 23(5): 9-12.

8. Castro Fernández J. En Costa Rica: ¿es impune la experimentación en seres humanos? Medicina Legal de Costa Rica 2002; 19(2): 103-116.

9. Ludovic R. Pruebas clínicas en seres humanos ;cuál es el límite? (Interview). LATINREC Bulletin 2007. (Sitio en Internet) Disponible en http://www.aislac.org/pdf/boletin_correoais/EntrevistaBol-ensayosclinicos.pdf Último Acceso en enero 29 de 2008.

10. Caniza M, Clara W, Maaron G, et al. Establishment of ethical oversight of human research in El Salvador: Lessons learned. The Lancet Oncology 2006; 7: 1027-1033.

11. Consuelo M, Palma G, Jaramillo E. Comités de ética de investigación en humanos: el desafio de su fortalecimiento en Colombia. Biomédica 2006; 26: 138-144.

12. Mancini Rueda R. Perspectiva Latinoamericana y del Caribe en Etica de la Investigación (Sitio en Internet) Disponible en http://www.paho.org/Spanish/BIO/Mancini.ppt Último Acceso en enero 29 de 2008.

13. De Young K, Nelson D. Latin America is Ripe for Trials and Fraud. Washington Post, December 21 2000, Seccion A, Pagina 01. (Sitio en Internet) Disponible en http://www.washingtonpost.com/ac2/wp-dyn/A31027-2000Dec20?lang uage=printer Último Acceso en febrero 12 de 2008.

14. Outomuro D. Reflexiones sobre el estado actual de la Ética de la Investigación en Argentina. Acta Bioethica 2004; 10(1): 81-94.

15. Lolas F. Ética de la publicación médica: legalidad y legitimidad. Acta Bioethica 2000; 6(2): 285.

16. Braunschweiger P, Goodman KW. The CITI Program: An International Online Resource for Education in Human Subjects Protection and the Responsible Conduct of Research. Academic Medicine 2007; 82: 861-864.

Recibido: 15 de febrero de 2008

Aceptado: 28 de febrero de 2008 